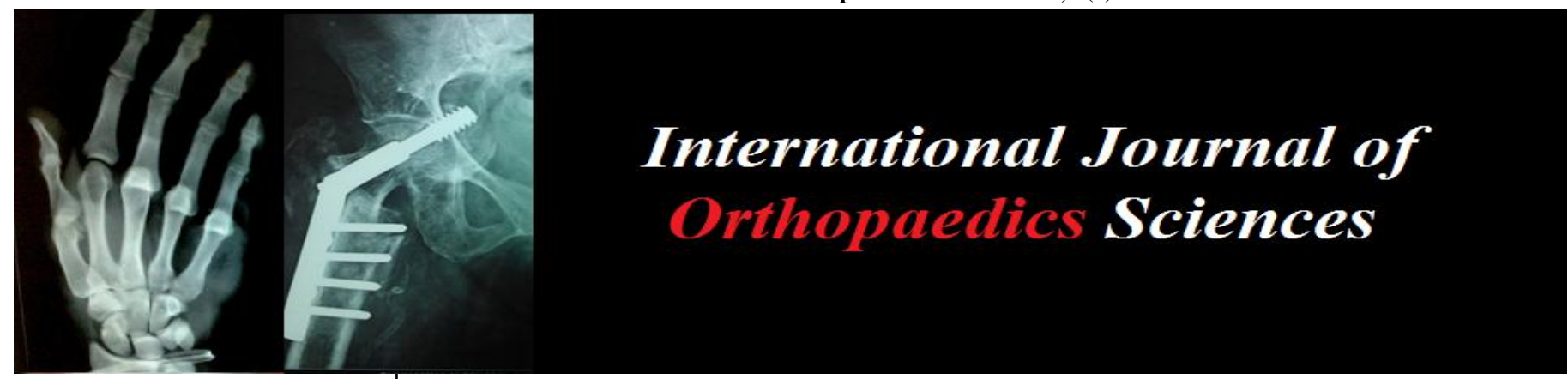

E-ISSN: 2395-1958

P-ISSN: 2706-6630

IJOS 2022; 8(1): 388-391

(C) 2022 IJOS

www.orthopaper.com

Received: 05-11-2021

Accepted: 07-12-2021

Mohammed Imran Basha DNB Orthopedic Surgery, Suwayr General Hospital (Al Jouf), AL Aziziya District,

Sakaka Al Jouf, Saudi Arabia

Corresponding Author: Mohammed Imran Basha DNB Orthopedic surgery, Suwayr general hospital (Al jouf), AL Aziziya District, Sakaka Al Jouf, Saudi Arabia

\section{Comparative study of arthroscopic and MRI findings of injured Knee}

\section{Mohammed Imran Basha}

DOI: $\underline{\text { https://doi.org/10.22271/ortho.2022.v8.i1f.3044 }}$

\section{Abstract}

Background: Knee joint is one of the most commonly injured joints because of its anatomical structure, its exposures to external forces and the functional demands placed on it. Because of the difficulty of obtaining an accurate clinical examination in the acute setting, the incidence of such injuries is not well understood.

Objectives: To Compare Arthroscopic and MRI findings of Injured knee.

Methods: 30 Patients who reported with knee symptoms after knee injury, who were indicative of Internal derangement and underwent arthroscopy procedure following a detailed clinical examination and imaging with MRI

Results: Based on Clinical examination, ACL injury was seen in $50 \%$ of the cases, Medial meniscus tear was seen in $26.66 \%$ of the cases and lateral meniscus tear in $16.66 \%$ of the cases. Based on MRI, ACL injury was seen in $63.33 \%$ of the cases, Medial meniscus tear was seen in $50 \%$ of the cases and lateral meniscus tear in $30 \%$ of the cases. Based on Arthroscopy, ACL injury was seen in $76.66 \%$ of the cases, Medial meniscus tear was seen in $36.66 \%$ of the cases and lateral meniscus tear in $16.66 \%$ of the cases. The accuracy was $100 \%$ for ACL tear and Osteochondral defect was $100 \%$. The accuracy was $86 \%$ for PCL and meniscal tears. The accuracy for lateral meniscal tears was $83 \%$.

Conclusion: Arthroscopy is gold standard and finer when compared to MRI in the diagnosis of meniscal and cruciate ligament tears. MRI is a useful non-invasive modality having moderate sensitivity, specificity and accuracy in the diagnosis of menisci and cruciate ligament injuries.

Keywords: Arthroscopy, MRI, knee injuries, ligament tear

\section{Introduction}

In present era, road traffic accidents and sports activities are the most prevalent causes of knee injuries. Injury to the ligaments and meniscus compromises the stability and normal mechanics of the knee joint, resulting in an unstable knee that makes it difficult for a person to carry out their routine activities ${ }^{[1]}$. As a result, these injuries should be accurately diagnosed and treated (surgically or conservatively) promptly. The most prevalent of these injuries is an ACL or a combination of ACL and meniscal injury ${ }^{[2]}$.

Radiography, CT scans for fractures, and MRI for soft tissue injuries in the knee joint are some of the imaging modalities employed to evaluate the knee. Arthroscopy is a procedure that can be used to diagnose and treat a joint ${ }^{[3]}$.

The initial evaluation technique utilised for every patient is a clinical examination. Because inspection of the joint may be impossible in acute instances due to discomfort and swelling, MRI has been reported in recent years to increase diagnostic precision without the use of ionising radiation ${ }^{[4]}$. It is non-invasive, consistent, and has several advantages over invasive diagnostic arthroscopy. As a result, the patient's morbidity is reduced.

However, there have been myriad disagreements about comparing MRI and clinical examination findings with arthroscopic findings of the knee joint ${ }^{[5]}$.

The goal of this study was to evaluate the effectiveness of clinical examination and MRI findings in knee joint injuries to arthroscopy, which is being used as the gold standard. The study's findings will lead to an early identification of the patient's injuries, allowing for prompt treatment and alleviation for the patient. 


\section{Materials and Methods}

Type of study: Randomized Prospective cross-sectional study.

Study setting: Suwayr General Hospital (Al Jouf)

Study Duration: April 2021 to September 2021

Study sample size: 30 Patients who reported with knee symptoms after knee injury, who were indicative of internal derangement and underwent arthroscopy procedure following a detailed clinical examination and imaging with MRI were the subject of the study.

\section{Inclusion Criteria}

- Patients aged between 21 to 50 yrs.

- Patients with an acute knee pain and suspicious knee injury, recent problems of locking of knee or effusion.

\section{Exclusion Criteria}

- Patients with severe comorbidities

- Patient with cardiac pacemakers, metal implants.

- Severe osteoarthritis of knee and Knee ankylosis.

\section{Methodology}

Thirty patients with a history of knee trauma who were admitted and treated at the Institute were included. All of the patients that were admitted were clinically examined and their injuries were documented. An X-ray of the knee joint was taken on a regular basis to check for any fractures. All patients with clinical symptoms suggesting internal derangement of the knee joint underwent an MRI.

Signs of injuries to the menisci, cruciate ligaments, collateral ligaments, articular cartilage, loose bodies, meniscal cysts, and bone contusions, as well as evidence of soft tissue injuries surrounding the knee joint, were examined using MRI scans. These patients were then taken for arthroscopy, both diagnostic and therapeutic. The diagnostic arthroscopy findings were documented.

The pathological process was depicted through well drawn illustrations while the operation procedure was carried out. For easy reference, these were attached to the patient's record. Photographs and videos were taken, and they were saved for later usage.

The operation theatre results comprised a review of the complete joint and anatomical structure, lesions associated with the existence or absence of a tear, its position, and the state of the articular cartilage, among other aspects. The composite data was compiled and compared with the results of the MRI.

\section{Statistical Analysis}

SPSS 22 software was used for statistical analysis. The data was presented in the form of tables. The sensitivity, specificity, positive predictive value (PPV) and the negative predictive value (NPV), in order to assess the reliability of the MRI results.

\section{Observation and Results}

Table 1: Distribution based on various demographics and parameters

\begin{tabular}{|c|c|c|}
\hline Gender & Number of cases & Percentage \\
\hline Male & 24 & $80 \%$ \\
\hline Female & 6 & $20 \%$ \\
\hline Age Group & & \\
\hline $21-30$ & 12 & $40 \%$ \\
\hline $31-40$ & 10 & $33.33 \%$ \\
\hline $41-50$ & 8 & $26.66 \%$ \\
\hline Side & & $80 \%$ \\
\hline Right knee & 24 & $20 \%$ \\
\hline Left knee & 6 & \\
\hline Mode of injury & & $40 \%$ \\
\hline $\begin{array}{c}\text { Motor vehicle } \\
\text { accident }\end{array}$ & 12 & $13.33 \%$ \\
\hline Sports Injury & 4 & $26.66 \%$ \\
\hline Domestic falls & 8 & $20 \%$ \\
\hline Others & 6 & \\
\hline
\end{tabular}

Male predominance was seen with $80 \%$ and females were 20\%. The male: female ratio was $4: 1$. The majority of the patients belonged to the age group of 21 to 30 yrs with $40 \%$, followed by 31 to $40 \mathrm{yrs}$ with $33.33 \%$ and 41 to $50 \mathrm{yrs}$ age group with $26.66 \%$ of the cases. The mean age group was $33.45 \pm 3.45$ yrs.

In $80 \%$ of the cases right knee was affected and in the rest $20 \%$ of the cases left knee was affected.

In majority of the cases around $40 \%$, The mode of injury was Motor Vehicle accident, followed by Domestic falls in $26.66 \%$ of the cases, Other causes in $20 \%$ of the cases and Sports injury in $13.33 \%$ of the cases.

Table 2: Distribution based on Injury to Structures

\begin{tabular}{|c|c|c|c|}
\hline Structures injured & Clinical Exam & MRI & Arthroscopy \\
\hline ACL & 15 & 19 & 23 \\
\hline PCL & 0 & 0 & 0 \\
\hline Medial meniscus & 8 & 15 & 11 \\
\hline Lateral meniscus & 5 & 9 & 5 \\
\hline
\end{tabular}

Based on Clinical examination, ACL injury was seen in $50 \%$ of the cases, Medial meniscus tear was seen in $26.66 \%$ of the cases and lateral meniscus tear in $16.66 \%$ of the cases.

Based on MRI, ACL injury was seen in $63.33 \%$ of the cases, Medial meniscus tear was seen in $50 \%$ of the cases and lateral meniscus tear in $30 \%$ of the cases.

Based on Arthroscopy, ACL injury was seen in $76.66 \%$ of the cases, Medial meniscus tear was seen in $36.66 \%$ of the cases and lateral meniscus tear in $16.66 \%$ of the cases.

Table 3: Distribution based on Ligament and meniscal tears.

\begin{tabular}{|c|c|c|c|c|}
\hline \multirow{2}{*}{ Tear } & \multirow{2}{*}{ MRI } & Positive & Arthroscopy & \multirow{2}{*}{ Total } \\
\cline { 2 - 5 } & & 19 & 0 & 19 \\
\hline $\begin{array}{c}\text { Anterior Cruciate Ligament } \\
\text { (ACL) Tears }\end{array}$ & Positive & 4 & 7 & 11 \\
\cline { 2 - 5 } $\begin{array}{c}\text { Posterior Cruciate Ligament } \\
\text { (PCL) Tears }\end{array}$ & Negative & 0 & 0 & 0 \\
\cline { 2 - 5 } Medial Meniscal tear & Positive & 0 & 40 & 30 \\
\cline { 2 - 5 } & Pegative & 0 & 15 & 15 \\
\hline \multirow{2}{*}{ Lateral Meniscal tear } & Negative & 5 & 21 & 9 \\
\cline { 2 - 5 } & Positive & 0 & & 21 \\
\end{tabular}


- The sensitivity and specificity of MRI with respect to Arthroscopy in ACL tear is $82 \%$ and $100 \%$.

- The sensitivity and specificity of MRI with respect to Arthroscopy in PCL tear is $100 \%$ and $100 \%$ correspondingly.

- The sensitivity and specificity of MRI with respect to arthroscopy in medial meniscal tears is $100 \%$ and $78 \%$ correspondingly

- The sensitivity and specificity of MRI with respect to arthroscopy for lateral meniscal tears is $100 \%$ and $84 \%$ correspondingly.

Table 4: Distribution based on Sensitivity, specificity and Accuracy of MRI

\begin{tabular}{|c|c|c|c|}
\hline Structure & Sensitivity & Specificity & Accuracy \\
\hline ACL & $82 \%$ & $100 \%$ & $86 \%$ \\
\hline PCL & $100 \%$ & $100 \%$ & $100 \%$ \\
\hline Medial meniscus & $100 \%$ & $78 \%$ & $86.66 \%$ \\
\hline Lateral meniscus & $100 \%$ & $84 \%$ & $83.33 \%$ \\
\hline Osteochondral defect & $100 \%$ & $100 \%$ & $100 \%$ \\
\hline
\end{tabular}

The accuracy was $100 \%$ for ACL tear and Osteochondral defect was $100 \%$. The accuracy was $86 \%$ for PCL and meniscal tears. The accuracy for lateral meniscal tears was $83 \%$.

\section{Discussion}

Knee joint injuries are becoming more prevalent. The necessity to correctly diagnose knee injuries is critical for optimal care and prognosis; otherwise, the patient would suffer from persistent debility ${ }^{[6]}$.

Arthroscopy has remained the gold standard for the diagnosis of internal derangements of the knee across many studies, against which other diagnostic modalities should be compared. The MRI's sensitivity, specificity, accuracy, positive predictive value, and negative predictive value were calculated in order to determine if it was possible to avoid MRI and do an arthroscopy in suspected cases after a clinical examination ${ }^{[7]}$.

Furthermore, arthroscopy allows for a full examination of the meniscus, ligaments, and cartilage through a keyhole. However, with non-invasive imaging such as MRI, a normal anatomic variance may appear to be a tear. This has an impact on the interpretation of MRI results in management ${ }^{[8]}$.

One can get a more precise conclusion and gain a better understanding utilising arthroscopy since it clearly characterises the nature and pattern of tear, whereas MRI can sometimes overdiagnose tears or diagnose typical anatomic variants as tears. In MRI studies, the exact pattern of the tear is not mentioned (radiologist dependant).

Because of the possibility of intra-observer and inter-observer variance in non-invasive imaging such as MRI, detecting a significant lesion is solely a radiologist's responsibility, which when misinterpreted in an MRI report might alter the whole treatment regimen. Diagnostic arthroscopy is required to avoid all of these misunderstandings and also serves as a gold standard for analysing the IDK lesion and, as a result, planning management.

Diagnostic arthroscopy may be performed in a small OT setup with minimal instrumentation, however therapeutic endoscopy requires more sophisticated instruments. MRI setup, on the other hand, necessitates considerable space and expenditure. The procedure's duration and cost are the two most significant disadvantages of routine MRI usage. Those patients who have cardiac pacemakers and have had acute knee injuries may find it uncomfortable. MRI setups can cause claustrophobia as well.

According to this study results, The most prevalent IDK complaint was pain and instability, whereas males were more likely to have knee injuries, with the right knee predominating. The most prevalent injury was an ACL tear, followed by a meniscal injury.

In comparison with arthroscopy, MRI has less specificity in detecting IDK. This can be attributed to the research's limitations, which include the fact that it is a non-randomized trial with a small number of patients. These observations, reflect the reality an orthopedic surgeon would encounter in their clinical practices. The accuracy, sensitivity and specificity values for knee lesions vary widely in literature. Rubin et al. reported $93 \%$ sensitivity for diagnosing isolated ACL tears ${ }^{[9]}$. Similarly several prospective studies have shown a sensitivity of $92-100 \%$ and specificity of $93-100 \%$ for the MR imaging diagnosis of ACL tears ${ }^{[10,11]}$.

Arthroscopy is favorable in cases when MRI is not effective, such as peripheral meniscal tears and inferior surface tears, since arthroscopy is more sensitive in detecting numerous meniscal tears that may be missed on MRI.

While MRI is less sensitive than arthroscopy in diagnosing ACL injuries, numerous anatomic variations on MRI might seem like tears.

In every patient with a suspected ligamentous injury, an MRI should not be performed, especially if the clinical signs are clear.

\section{Conclusion}

In concurrent knee injuries, MRI has a propensity to over diagnose or misinterpret a lesion, according to this study. When an MRI scan is normal, an arthroscopy should not be denied. Because of this limitation in MRI, it has been determined that arthroscopy may be performed after a complete clinical evaluation sans MRI.

In the diagnosis of meniscal and cruciate ligament injuries, arthroscopy is the gold standard and better than MRI. In the diagnosis of meniscal and cruciate ligament injuries, MRI is a good non-invasive method with intermediate sensitivity, specificity, and accuracy.

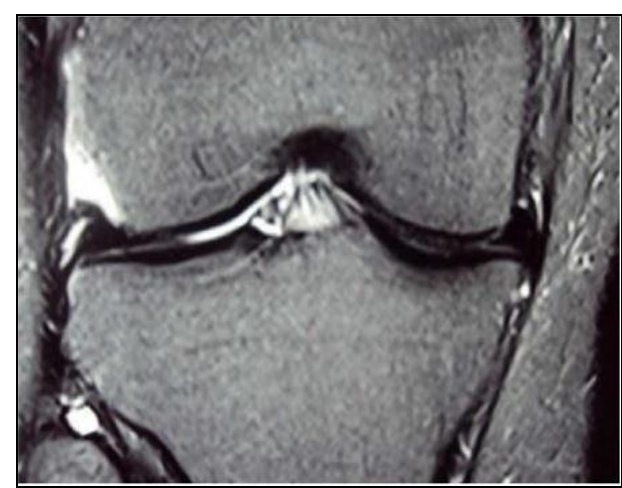

Fig 1: Medial meniscal tear- MRI

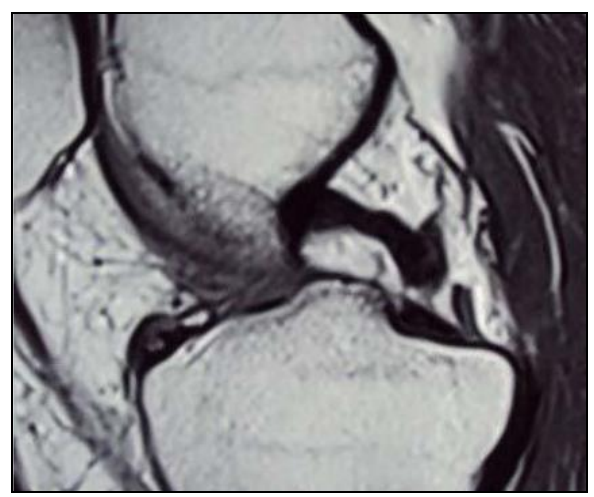

Fig 2: ACL tear - MRI 


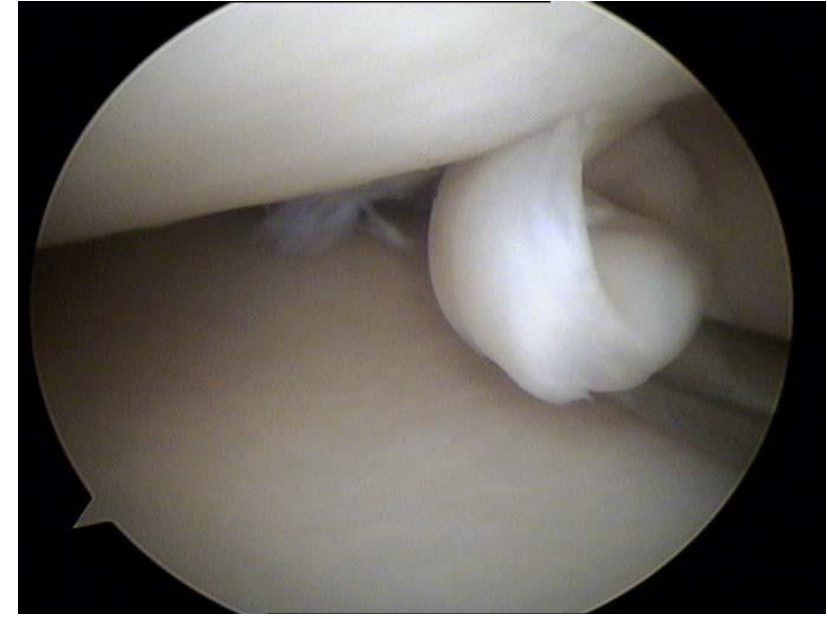

Fig 3: Medial meniscal tear - Arthroscopy

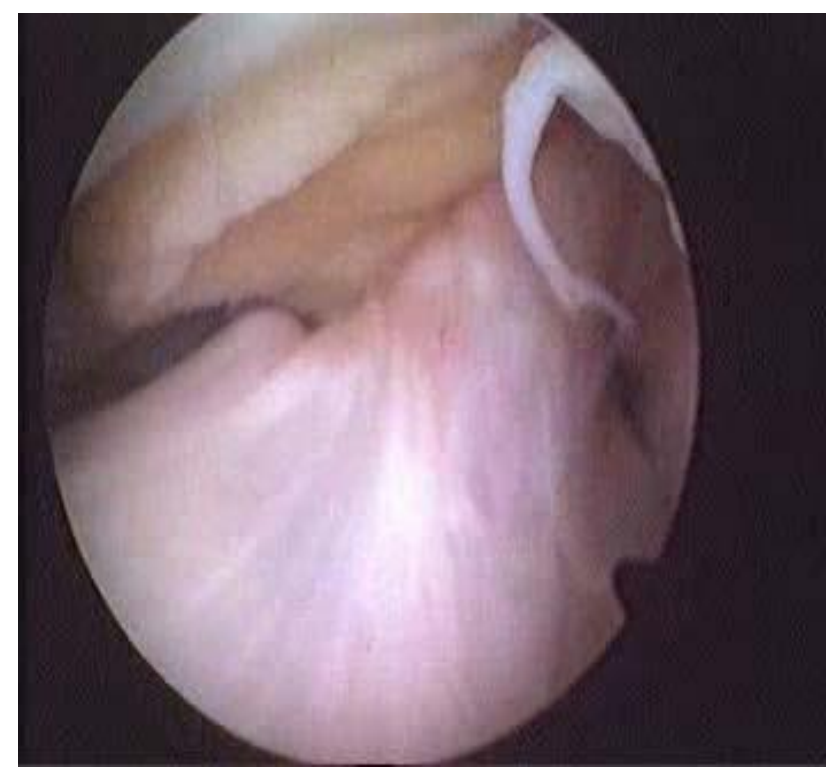

Fig 4: ACL tear - Arthroscopy

\section{References}

1. Kaplan PA, Walker CW, Kilcoyne RF, Brown DE, Tusek D, Dussault RG. Occult fractures patterns of the knee associated with ACL tears. Assessment with MR imaging. Radiology. 1992;183:835-8.

2. Gillquist J, Hagberg G, Oretorp N. Arthroscopic visualization of the posteromedial compartment of the knee joint. Orthop Clin North Am. 1979;10(3):545-7.

3. Avcu S, Altun E, Akpinar I, Bulut MD, Eresov K, Biren T. Knee joint examination by MRI:the correlation of pathology, age and sex. N Am J Med Sci. 2010;2(4):2024.

4. Esmaili Jah AA, Keyhani S, Zarei R, Moghaddam AK. Accuracy of MRI in comparison with clinical and arthroscopic findings in ligamentous and meniscal injuries of the knee. Acta Orthop Belg. 2005;71(2):18996.

5. Navali AM, Bazavar M, Mohseni MA, Safari B, Tabrizi A. Arthroscopic evaluation of the accuracy of clinical examination versus MRI in diagnosing meniscus tears and cruciate ligament ruptures. Arch Iran Med. 2013;16(4):229-32.

6. Mudhusudan TR, Kumar TM, Bastawrous SS, Sinha A. Clinical examination, MRI and arthroscopy in meniscal and ligamental knee injuries- a prospective study. Journal of Orthopaedic Surgery and Research. 2008;15:3-19.
7. Munshi M, Davidson M, MacDonald PB, Froese W, Sutherland K. The efficacy of magnetic resonance imaging in acute knee injuries. Clin J Sport Med. 2000;10:34-9.

8. La Prade RF, Gilbert TJ, Bollom TS, Wentorf F, Chaljub $\mathrm{G}$. The magnetic resonance imaging appearance of individual structures of the posterolateral knee. A prospective study of normal knees and knees with surgically verified grade III injuries. Am J Sports Med. 2000;28:191-199.

9. Rubin DA, Kettering JM, Towers JD, Britton CA. MR imaging of the knee having isolated and combined ligament injuries. AJR. 1998;170:1207-1213.

10. Kumar S, Bansal A. A Comparative Study of Accuracy of Clinical Examination, MRI \& Arthroscopy Findings in Cruciate Ligament and Meniscal Injuries of the Knee. Indian Journal of Basic \& Applied Medical Research. 2011;1(1):106-113.

11. Noha Behairy $\mathrm{H}$, Mohsen Dorgham A, Sherif. AK. Accuracy of routine MRI in meniscal and ligamentous injuries of the knee: comparision with arthroscopy. Int Orthop SICOT. 2009;33(4):961-7. 